\title{
A Simple Method to Test Microscope Objectives Using a Laser Pointer
}

\section{Thomas Hanscheid}

Instituto de Microbiologia, Faculdade de Medicina, Universidade de Lisboa, Avenida Prof. Egas Moniz, 1649-028 Lisboa, Portugal t.hanscheid@medicina.ulisboa.pt

\begin{abstract}
The objective lens is regarded as the critical component for an optimal image in light microscopy. Not every damage to the lens surface may be easily visible during maintenance when the objective is removed and observed. The internal lens organization produces a magnified image of the outer lens surface. Shining the light of a laser pointer into the lens, and reproducing the image of the light exiting onto a flat surface, produces different images for intact and damaged objective lenses.
\end{abstract}

Keywords: light microscope, maintenance, objective lens, damaged lens, laser pointer

\section{Introduction}

Light microscopes are widely used in research, routine laboratories, and teaching, and they often represent a substantial investment. Today many different types of microscopes exist, but they all have in common that the objective lens is regarded as the critical component for an optimal image [1]. The need for correction of optical aberrations is the main driver of the difference between low-cost objective lenses, as used by basic student microscopes, and the much more expensive objectives for advanced research microscopes. Microscopes are optical instruments which require regular maintenance, especially cleaning dirt and dust from optical surfaces along the light path $[2,3]$. The front lens of the objective is especially sensitive to dust and dirt, which can result in a markedly reduced resolution and suboptimal images. The price of sophisticated instruments, such as confocal or spinning disk microscopes, justifies professional maintenance, usually from the manufacturer. On the other hand, especially in teaching, limited budgets dictate that it is often the in-house staff that performs maintenance procedures. For this second scenario a series of excellent publications give very helpful and practical, step-by-step guidance, even if they do not all agree on all aspects, such as the recommended type of solvents that should be used to remove immersion oil or other fat from the lens [1-6]. This article describes a lens inspection procedure employing a common laser pointer.

\section{Materials and Methods}

Objective lenses should be inspected after each use, and oil should be removed form immersion objectives. Standard operating procedures (SOPs) call for regular removal of the objective and careful inspection using a stereo dissection microscope or, as an alternative, a magnifying glass or an inverted eyepiece [2-4]. This allows light from a window or lamp to reflect off the lens, which reveals most irregularities on the surface. The lens should be clear and free of oil, dust, or other particles. One should screen for scratches on the surface or adherent contaminations. The lens seal should be checked for damage, which might have allowed oil to seep inside [2]. Interestingly, examining the rear aperture of the objective is supposed to help identify dirt on the external lens surface because the internal lens organization produces a magnified image of these contaminations [5].

The frequent removal of oil from an immersion lens at the end of each session is likely to have a high propensity to be performed poorly or even wrongly, especially where novices may be at work, as in undergraduate teaching labs. The use of inadequate products, for example, abrasive tissues) or by employing an incorrect technique (for example, applying excessive direct pressure in a zig-zag pattern) has a high potential to damage the lens surface $[1,5]$. Importantly, while this may produce a degraded image quality, such as hazy images, the damage to the glass itself may not be immediately obvious and may be difficult to detect [2].

In our institution 25 light microscopes are used in practical classes to teach clinical microbiology to undergraduate students (Figure 1A). They are equipped with a 100×, achromat, oil-immersion objective (Figure 1B). Using a routine blood smear from a patient with malaria (Figure 2A), the quality of images is assessed at the end of term, as part of our maintenance and cleaning tasks.

\section{Results}

One microscope produced a very poor, hazy image (Figure 2B). The quality of the remaining optical pathway was checked by swapping the objective with another microscope, which confirmed that the cause of the poor resolution was, indeed, the objective. The external lens was thoroughly cleaned, including the use of aggressive solvents, such as xylene and ether, without any improvement in the image. Inspection of the lens using an inverted eyepiece and a stereomicroscope did not reveal any apparent damage.

Based on the idea that any surface changes should be magnified at the reverse aperture [5], the light of a common laser pointer was used to test this. The front lens of the objective

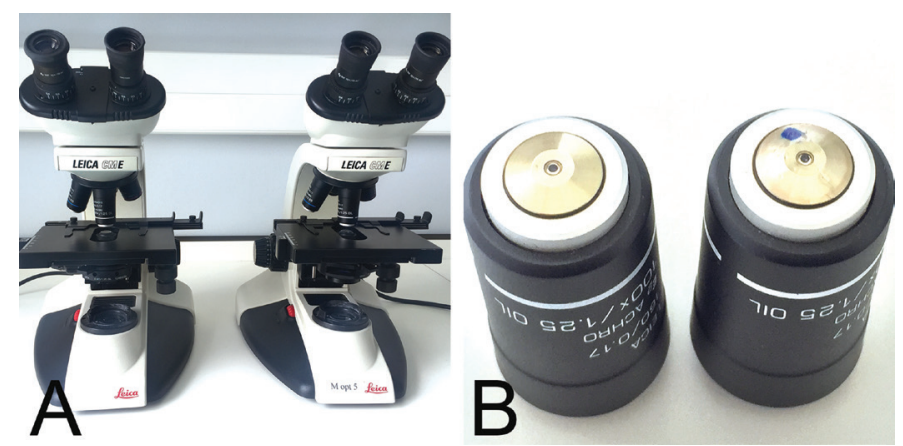

Figure 1: Microscope and objective. Leica light microscope, model CME, for student teaching $(A)$, with $100 \times / 1.25$, achromat, oil immersion objectives $(B)$. $A$ normal functioning objective is shown on the left and a damaged objective (blue dot) on the right. 


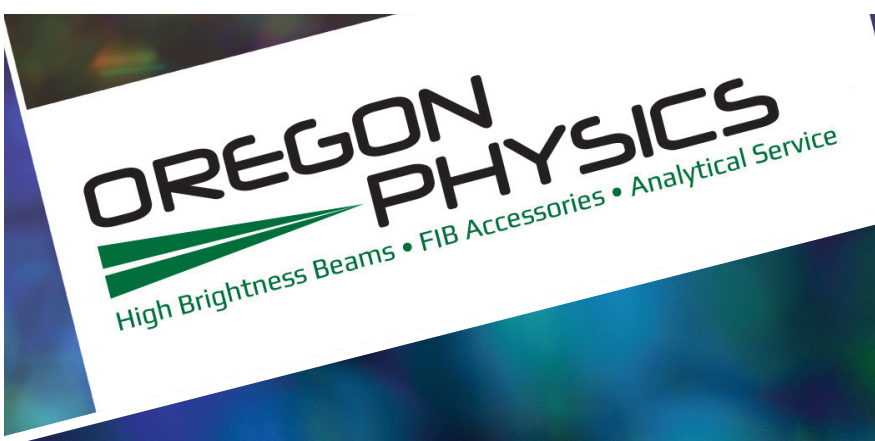

\section{Reduce Your FIB Operating Costs}

Managing multiple tools means maintaining system uptime while also saving costs. Let us help with replacement aperture strips, extractors and suppressors.

Oregon Physics has been making FIB consumable parts for over 12 years. Our parts meet and even exceed OEM quality standards so you can order with confidence.

Aperture strips can be made to standard or custom specifications.

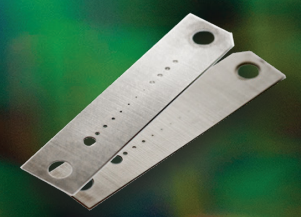

\begin{tabular}{|c|c|}
\hline $\begin{array}{l}\text { For FEI/Thermo Fisher } \\
\text { Scientific FIB Columns: }\end{array}$ & Discounts for: \\
\hline Phoenix & \multirow{5}{*}{$\begin{array}{l}\text { - } 10 \text { or more each } \\
\text { - } 5 \text { or more part sets } \\
\text { - Increased discounts } \\
\text { start at } 20 \text { units. }\end{array}$} \\
\hline Tomahawk & \\
\hline Sidewinder & \\
\hline Magnum & \\
\hline Pre-Lens & \\
\hline
\end{tabular}

We offer regularly scheduled deliveries and fast turnaround for urgent requests.

Contact us to arrange a consumables replacement program to best fit your needs.

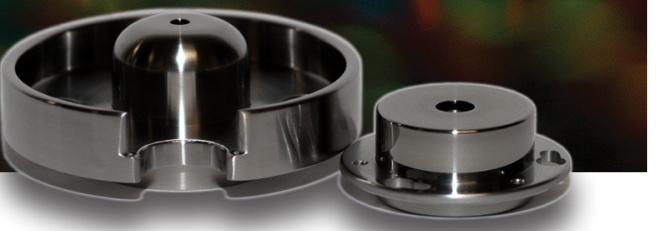

+15036010041

info@oregon-physics.com alluxa.com

\section{OPTICAL COATINGS REDEFINED}

\section{YOUR OPTICAL COATING PARTNER}

www.oregon-physics.com 


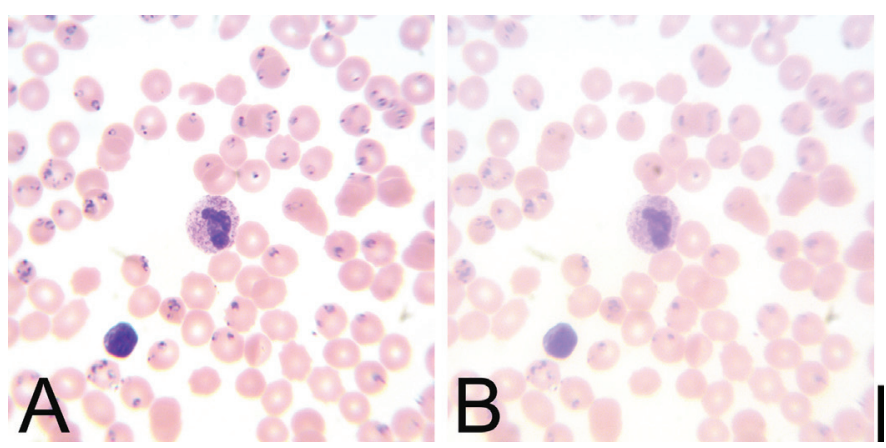

Figure 2: Blood smear to assess microscope image. Routine blood smear from a patient with malaria showing numerous parasitized erythrocytes $(50 \%$ parasitemia), one lymphocyte (bottom left), and one granulocyte (center). The same field of view was imaged using the same microscope after exchanging the objectives shown in Figure 1B: Normal image $(A)$ and hazy image with damaged objective (B).

was aligned with the laser beam of the pointer (laser pointer JD-303, $532 \mathrm{~nm}, 20 \mathrm{~mW}$; supplied by Alibaba), and the light exiting at the reverse aperture of the objective was projected onto a flat surface at a distance of half a meter (Figure 3). The image of an undamaged objective produces a normal image that shows a rather homogenous green projection (Figure 3A). The objective producing the poor image (Figure 3B), however, reveals markedly different patterns, with circular black rings and dark irregular areas, which move with the rotation of the objective. Using this technique with other objectives demonstrated that dirt or particles usually show up as well-defined, small, isolated dark spots, which disappear after cleaning.

The exact nature of this effect is not completely clear, but likely the coherent, polarized light of the laser may be more sensitive to minute damage on the lens surface compared to natural light or light from a common room lamp. Laser pointers use an inexpensive diode laser and are available for 10-20 US\$. This method could prove to be a simple and inexpensive way to check objective lenses for damage. Using a neutral-density (ND) filter and a common camera, which can be connected to a laptop, one can imagine the construction of a cheap device that would allow use of image analysis to provide results for this test (Figure 4).
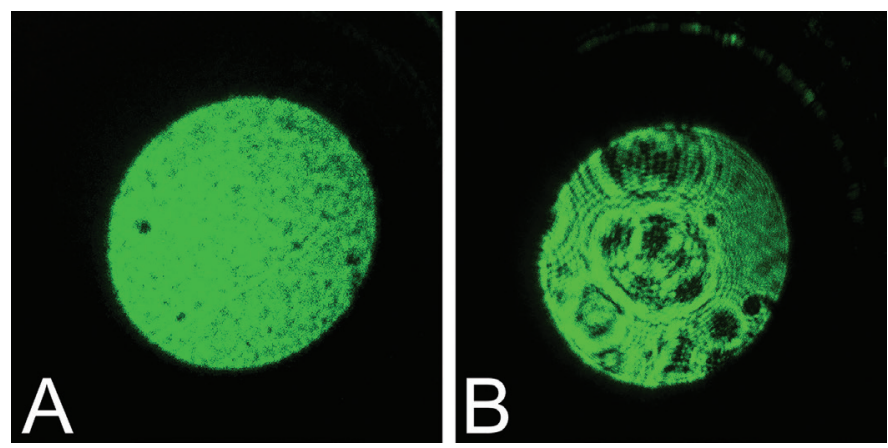

Figure 3: Projected image using a laser pointer. A common $20 \mathrm{~mW}, 532 \mathrm{~nm}$ laser pointer used for teaching was placed directly in front of the external lens of the objective, and the light exiting the reverse aperture was projected on a white wall, producing the images from a normal functioning objective $(A)$ and from the damaged objective (B). Note that the white wall appears black because the image is underexposed due to the intensity of the laser light.

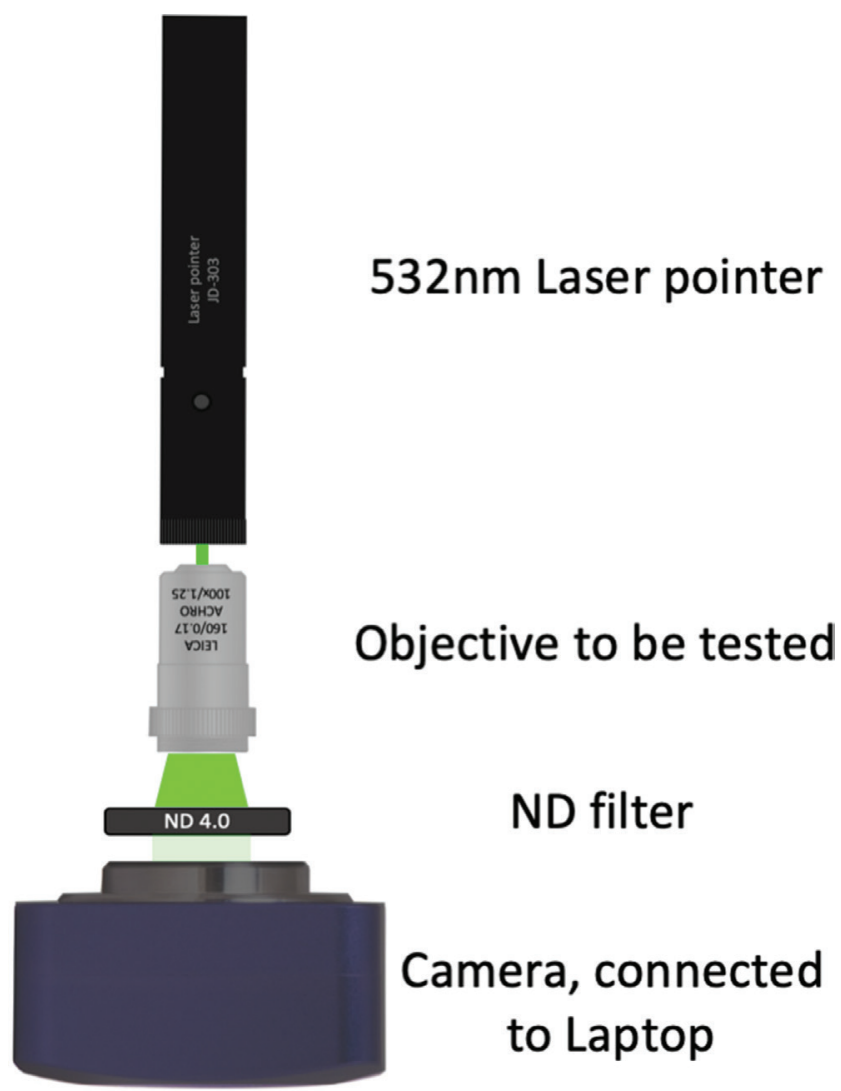

Figure 4: Possible apparatus to measure the observed effect. Using a laser pointer (or only a laser diode), an appropriate neutral-density (ND) filter to avoid over-exposure at the camera, and a microscope (ocular) camera or similar. This setup would allow capture of images with simple imaging software, such as ImageJ (https://imagej.nih.gov/ij/features.html, accessed Aug. 7, 2019). The assumption would be that a perfect lens surface should produce an image where all pixels show equal values.

\section{Conclusion}

A laser pointer can be used to detect dirt and damage in light microscopy objective lenses. When cleaning and visual inspection fail to show any irregularity in a poor performing objective, a simple test with a laser pointer revealed damage unnoticed by other means.

\section{References}

[1] ST Ross et al., Methods Cell Biol 123 (2014) 19-34.

[2] LJ Petrak and JC Waters, Methods Cell Biol 123 (2014) $55-76$.

[3] RC Deagle et al., Int J Biochem Cell Biol 89 (2017) 120-24.

[4] B Eason et al., "Microscope maintenance and quality control: a practical guide" in Microscopy: Advances in Scientific Research and Education, vol. 2., ed. A Méndez-Vilas, Formatex Research Center, Badajoz, Spain, 2014, 713-24.

[5] R Rottenfussser et al., "Microscope cleaning and maintenance, Education in Microscopy and Digital Imaging," Zeiss, http://zeiss-campus.magnet.fsu.edu/articles/basics/ care.html (accessed October 7, 2019).

[6] J James, Light Microscopic Techniques in Biology and Medicine. Martinus Nijhoff medical division, The Hague, The Netherlands, 1976, 133-40. 


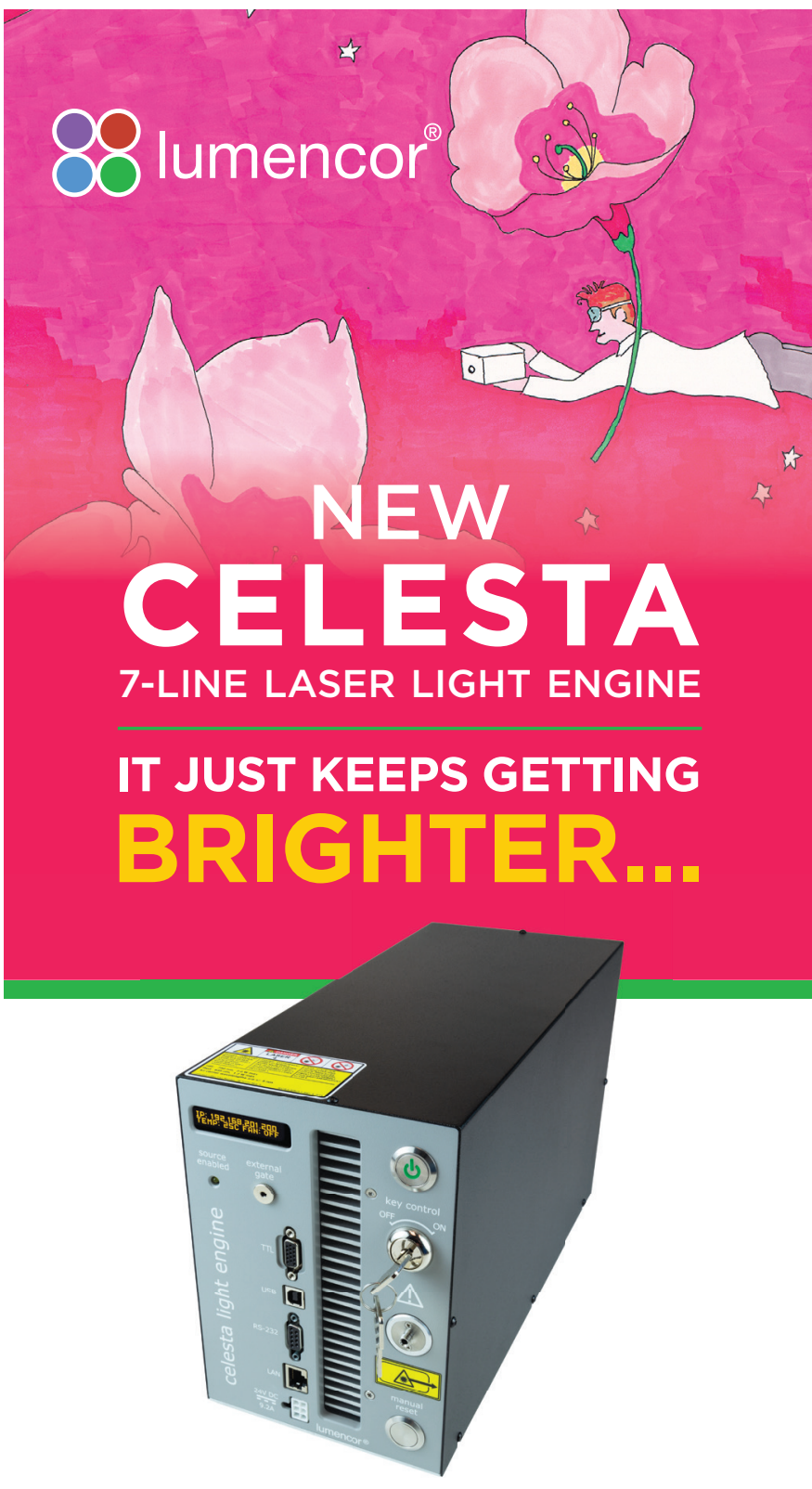

- Spectral breadth: 408, 445, 473, 518, 545 , 635, $750 \mathrm{~nm}$

- Power: 1000mW/color, 7W total

- Control: Active power stabilization

- Stability: Exceptional reproducibility, ideal for quantitation

- Ease of use: Pre-aligned, independent lasers

- Applications: Confocal, MERFISH, superresolution microscopy, photoactivation, optogenetics, FRAP, DNA-PAINT and more
Refurbishing or servicing an electron microscope? Scope us out for your ion pump needs.

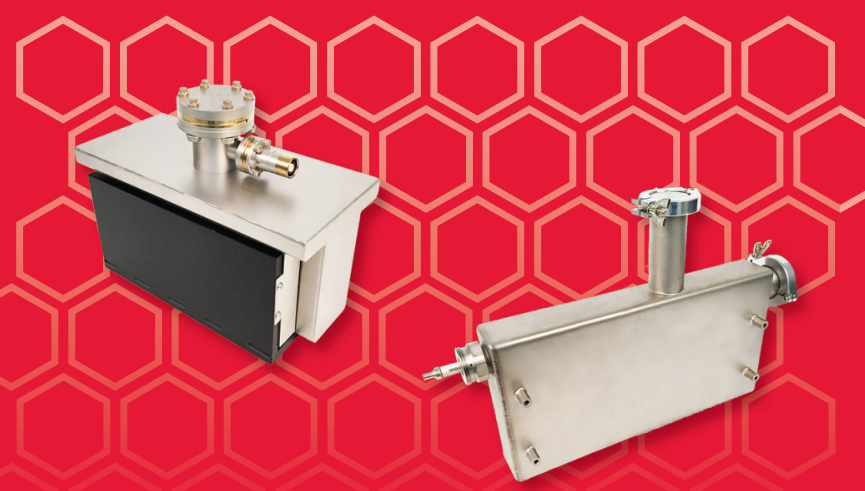

New | Rebuilt | Rebuilding Services DUUNIVMAM. 800.44E.8E11

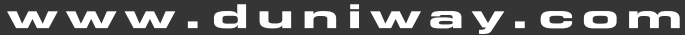

\section{MRS-6}

We are ISO-9000 certified and ISO-17025 accredited Microscopy Calibration Standard

Now you can calibrate better from $1,000 X$ to $1,000,000 X$ !

This is our fifth generation, traceable, magnification reference standard for all types (SEM, FESEM, Optical, STM, AFM, etc.) of microscopy. The MRS-6 has multiple $X$ and $Y$ pitch patterns ranging from $80 \mathrm{~nm}( \pm 3 \mathrm{~nm})$ to $2 \mu \mathrm{m}$ and 3 bar targets from $80 \mathrm{~nm}$ to $3 \mu \mathrm{m}$. There is also a STM test pattern. Definition of the $80 \mathrm{~nm}$ pitch pattern is excellent.

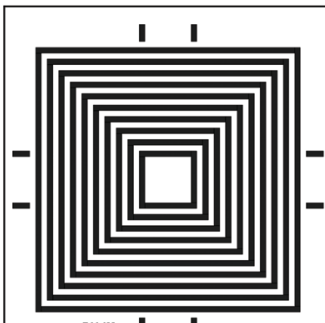

a $2 \mathrm{~m}$ 】 $\mathrm{PITCH}$

3BAR TARGETS

3H m

$\underset{\text { PITCH }}{3 \mu \mathrm{m}}=$

$\underset{\text { PITCH }}{2 \mu \mathrm{m}} \equiv \mathbf{I I}$

${ }_{\text {PITCH }}^{1.5 \mu \mathrm{m}} \equiv$ III $\underset{\text { PIICH }}{\text { PoOnm }} \equiv$ III $\underset{\text { PIICH }}{\text { Ponn }} \equiv$ III
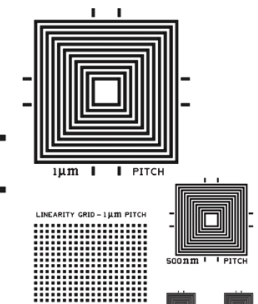


soonmerren $\equiv$

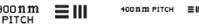

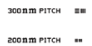
2000mm riren

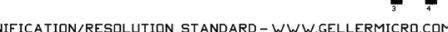
$S / N: M R S-6-2-$

\section{GELLER}

\section{MICROANALYTICAL} LABORATORY, Inc.

426e BOSTON ST., TOPSFIELD, MA 01983-1216 TEL: 978 887-7000 FAX: 978-887-6671 www.GellerMicro.com 